\title{
Una nueva especie de Hyptis (Labiatae) de Colombia
}

\author{
por \\ José Luis Fernández-Alonso \\ Real Jardín Botánico, CSIC, Plaza de Murillo 2, E-28014 Madrid, España. jlfernandeza@rib.csic.es
}

\begin{abstract}
Resumen
Fernández-Alonso, J.L. 2010. Una nueva especie de Hyptis (Labiatae) de Colombia. Anales Jard. Bot. Madrid 67(2): 127135.

Se describe e ilustra Hyptis jacobi Fern. Alonso, una nueva especie de porte arbustivo, conocida sólo de la cuenca media del río Chicamocha (departamento de Santander) en la vertiente occidental de la Cordillera Oriental de Colombia. Esta planta que tiene alguna afinidad con la especie andina $H$. tafallae (sección Umbellatae) es muy diferente de las especies de Hyptis conocidas previamente de Colombia.
\end{abstract}

Palabras clave: Hyptis, nueva especie, Chicamocha, Colombia, Hyptidendron, Labiatae, taxonomía.

\section{Introducción}

Las recientes expediciones botánicas de recolección enmarcadas en proyectos de bioprospección y taxonomía de Labiatae, que tuvimos oportunidad de realizar en algunos sectores poco explorados de la cuenca media del río Chicamocha (cañón del Chicamocha), sector Guaca-Molagavita-Málaga, permitieron localizar algunos táxones no descritos de asteráceas, labiadas y bombacáceas, que están siendo objeto de estudio y descripción en la actualidad. Sin duda uno de los más llamativos es la especie del género Hyptis Jacq. (Labiatae) que a continuación se describe.

El género Hyptis, el segundo en importancia de las labiadas americanas, está constituido por al menos 290 especies casi exclusivamente neotropicales (Epling, 1949; Harley \& al., 2004). Según los estudios recientemente realizados, Hyptis está representado en Colombia por 44 especies de hierbas y pequeños arbustos, incluyendo la especie arbustiva que ahora se describe (Fernández-Alonso, 1995). En Colombia es el segundo género más diverso de las Labiadas, después de Salvia L., que cuenta con 85 táxones reconocidos hasta la fecha en el territorio (Fernán-

\begin{abstract}
Fernández-Alonso, J.L. 2010. A new species of Hyptis (Labiatae) from Colombia. Anales Jard. Bot. Madrid 67(2): 127-135 (in Spanish).

The shruby new species, Hyptis jacobi Fern. Alonso, only known from the central Chicamocha valley (Department of Santander) in the western slope of the Cordillera Oriental of Colombia, is described and illustrated. This species is related to the Andine H. tafallae (Sect. Umbellatae) but is quite different from other Hyptis previously known from Colombia.
\end{abstract}

Keywords: Hyptis, new species, Chicamocha, Colombia, Hyptidendron, Labiatae, taxonomy.

dez-Alonso, 1995, 2008; Fernández-Alonso \& al., 2003).

Hyptis jacobi Fern. Alonso, sp. nov. (Figs. 1-4)

Tipo: Colombia. Santander: Vía Málaga-Bucaramanga, km 6-7 después de Málaga, Vereda Buenavista, 642'29” N 7245'26”W, 2670 m, 29-VI-2009, arbusto de $4 \mathrm{~m}$, con tallos escandentes y panículas terminales amplias, fl, fr, J.L. Fernández-Alonso 28193 \& C.N. Díaz-Pérez (holotypus, COL; isotypus, COL, G, HUA, K, MA, MO, UIS, US).

Affinis Hyptis tafallae (sectio Umbellatae) sed differt indumento caulis dense et molliter lanato-tomentoso, pilis ramosis, inflorescentiis paniculatis magnis, difussis, distico-complanatis, omne conspicue purpureo pilosis; cimulis terminalibus 2-3 floriferis (exumbellatis); dentes calycis longiores usque ad $2 \mathrm{~mm}$ longis et corollae tubo breviore, usque ad $3,5 \mathrm{~mm}$ longis.

Arbusto escandente 3,5-4,5(5) $\mathrm{m}$, poco ramificado, con ejes principales subcilíndricos, obscuramente tetrágonos, corteza grisáceo-pajiza, estriada o rugosa, con restos de recubrimiento lanoso-tomentoso (Fig. 2c), de 


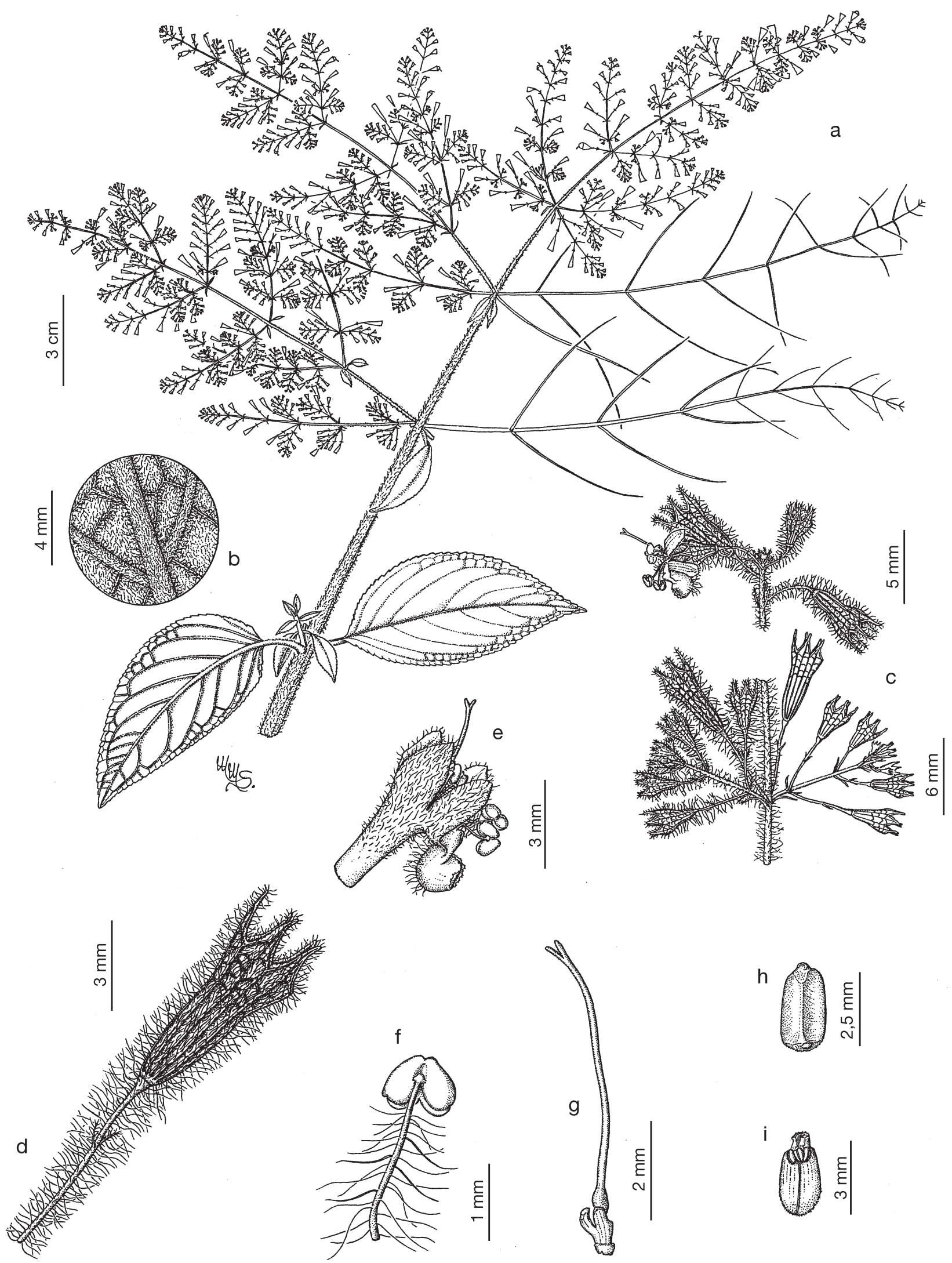

Fig. 1. Hyptis jacobi Fern. Alonso: a, detalle de un eje terminal de la planta, con una panícula; $\mathbf{b}$, detalle del indumento en el envés de la hoja; c, címulas mostrando la disposición de flores y bractéolas; d, pedicelo floral y cáliz; e, corola, mostrando la posición de las anteras y el estilo; $\mathbf{f}$, detalle de un estambre; $\mathbf{g}$, ginobase lobulada y estilo; $\mathbf{h}$, núcula en vista dorsal; i, núcula en vista ventral (dibujado a partir de Albesiano \& al. 2003, parátipos). 
3,5-5 cm de DAP; brotes jóvenes cubiertos por un indumento compacto de pelos ramificados, lanoso-algodonosos (Fig. 3b,c), color blanco-morado, que en seco se torna leonado o marrón; entrenudos 3,5-6 cm, más cortos que las hojas. Hojas con pecíolo 1,3-2,2 cm, 3-4 $\mathrm{mm}$ de grosor, densamente lanoso-tomentoso; limbo 7 $12 \times 3,5-6 \mathrm{~cm}$, color verde oscuro o verde-morado por el haz, con ondulaciones en la cara superior y color blanquecino o blanco-morado por el envés (Fig. 3); cartáceas y engrosadas; ovado-lanceoladas, agudas en el ápice y redondeadas o subcordadas, raramente cuneadas en la base; hojas basales de brotes jóvenes hasta de $20 \times 10 \mathrm{~cm}$, con base claramente cordada; senos o aurículas basales de 4-6 cm; margen foliar fina, irregular y doblemente crenulado; haz finamente bullada o ampollosa, con abundante indumento rizado de aspecto simple y envés densamente lanoso-blanquecino; con 7-11 pares de nervios laterales y con nerviación terciaria y cuaternaria reticulada y excavada en el haz e inconspicua por el envés. Inflorescencia en panículas terminales amplias, llamativamente moradas, marrones en la fructificación, hasta de $70 \times 40 \mathrm{~cm}$, difusas y de aspecto dístico (Figs. 1a, 2a,b), constituidas por 7-11 pares de panículas parciales que parten de la axila de hojas 2,5$3,5 \times 1,5-2 \mathrm{~cm}$, ligeramente reducidas, verdes, bulladas por el haz y tomentosas color blanco marrón por el envés; panículas parciales de hasta $30-40 \mathrm{~cm}$, con al menos dos grados de ramificación, dísticas, acompañadas de brácteas foliáceas muy reducidas; ejes de la inflorescencia densamente pilosos, con pelos 1,5-2,5 mm, patentes, morados, en seco amarillentos (tratados con alcohol). Flores agrupadas en címulas simples o ramificadas dicotómicamente (Fig. 4a,b), con 2-5 flores; pedúnculos de las címulas 2-3 mm, cortos, con brácteas lineares muy reducidas. Pedicelos florales gráciles, filiformes, articulados, $5-8(10) \mathrm{mm}$, iguales o ligeramente más largos que el cáliz (Fig. 1c), con dos bractéolas filiformes de 1-2 mm en la articulación; indumento color púrpura-morado, erecto, ligeramente rizado, de pelos simples de 1,5-2 mm. Cáliz 4,5-6 mm en la antesis, acrescente, de hasta 9,5 $\mathrm{mm}$ después de la floración (Fig. 4c), cilíndrico-acampanado, morado, con tubo cilíndrico, de 3,5-4,5 mm en la antesis y hasta de 6-7,5 $\mathrm{mm}$ en la madurez (Fig. 4d), ligeramente ensanchado en la boca, de c. $4 \mathrm{~mm}$ de diámetro, membranáceo, de aspecto reticulado; nervios con pelos simples, largos, llamativos, color púrpura; espacios entre los nervios glabros o glabrescentes; dientes 1,4-1,8(2,0) mm, iguales o subiguales, estrechamente triangular-deltoides, acuminados. Corola 5-6 mm, color morado, con indumento disperso, morado, de pelos simples de 1-1,5 $\mathrm{mm}$; tubo 3,2-3,5 mm, cilíndrico, recto o ligeramente ensanchado en la parte distal; labio superior profunda- mente bilobado, con lóbulos de 1,5 mm; labio inferior trilobulado con lóbulos laterales c. $2 \mathrm{~mm}$, oblongos, erectos, y lóbulo medio 1,7-2 mm de diámetro, cuculado, recurvado-retrorso, hemisférico. Estambres 4 (Fig. 1e,f), que igualan o rebasan ligeramente en 2-3 $\mathrm{mm}$ los lóbulos de la corola; filamentos $2,5-3 \mathrm{~mm}$, con pelos 1-1,3 mm, largos, llamativos; anteras 0,8-1,0 mm de anchura, reniformes o deltoides. Ginobase nectarífera marcadamente bilobulada (Fig. 1g), que sobrepasa en longitud a los cuatro lóbulos del ovario (primordios de núculas), ubicadas lateralmente; por lo general solo 1-2(3) desarrolladas y (1)2-3 abortadas, según las flores. Estilo 6,5-7 mm, recto, que sobrepasa ligeramente los estambres y los lóbulos de la corola, glabro, ligeramente ensanchado en su base, y cortamente bifurcado en el ápice. Núculas 2,5-3 × 1,4-1,8 mm, nítidas, rectangular-oblongas, aplanadas y adelgazadas lateralmente, color marrón oscuro, con leve costilla longitudinal central, dorsiventral, con extremo apical, adelgazado, con una zona excavada; testa fina y dispersamente tuberculada, ligeramente glutinosa, desprovista de mucílago (Fig. 1h,i).

Etimología: Una especie tan destacada de mastranto colombiano me pareció indicada para recordar al maestro y amigo Santiago Castroviejo Bolibar, que tanto hizo por el estudio de la flora ibérica y americana.

\section{Hábitat, distribución y conservación}

Hyptis jacobi se conoce en la actualidad sólo de un pequeño sector de la Cordillera Oriental de Colombia (vertiente occidental), ubicado en la cuenca media del río Chicamocha, entre los 2200 y los $2700 \mathrm{~m}$ de altitud. Esta franja de bosque andino se encuentra en la actualidad muy perturbada por el antiguo establecimiento de cultivos y potreros para el ganado vacuno. Aunque no se descarta la presencia de esta planta en ambiente de borde de bosque de roble (Quercus bumboldtii Bonpl.), en sectores mejor conservados de la cuenca, hasta la fecha sólo ha sido observado en ocho puntos, siempre en borde de finca, formando parte de cercas vivas y pequeños remanentes de matorral asociado a pendientes rocosas. En este ambiente se asocia a Alnus acuminta Kunth, Lippia birsuta L. f., Lepechinia bullata (Kunth) Epling, Baccharis mutisiana Cuatrec., Passiflora mollissima (Kunth) L.H. Bailey, Salvia rubescens Kunth subsp. dolychothryx J.R.I. Wood \& Harley y a otras especies de Asteraceae (Ageratina Spach., Baccharis L., Pentacalia Cass., Stevia Cav.), Myricaceae (Morella Lour.), Myrsinaceae (Myrsine L.), Onagraceae (Fuchsia L.), Orchidaceae (Epidendrum L., Maxillaria Ruiz \& Pav.), Oxalidaceae (Oxalis L.), Pteridó- 

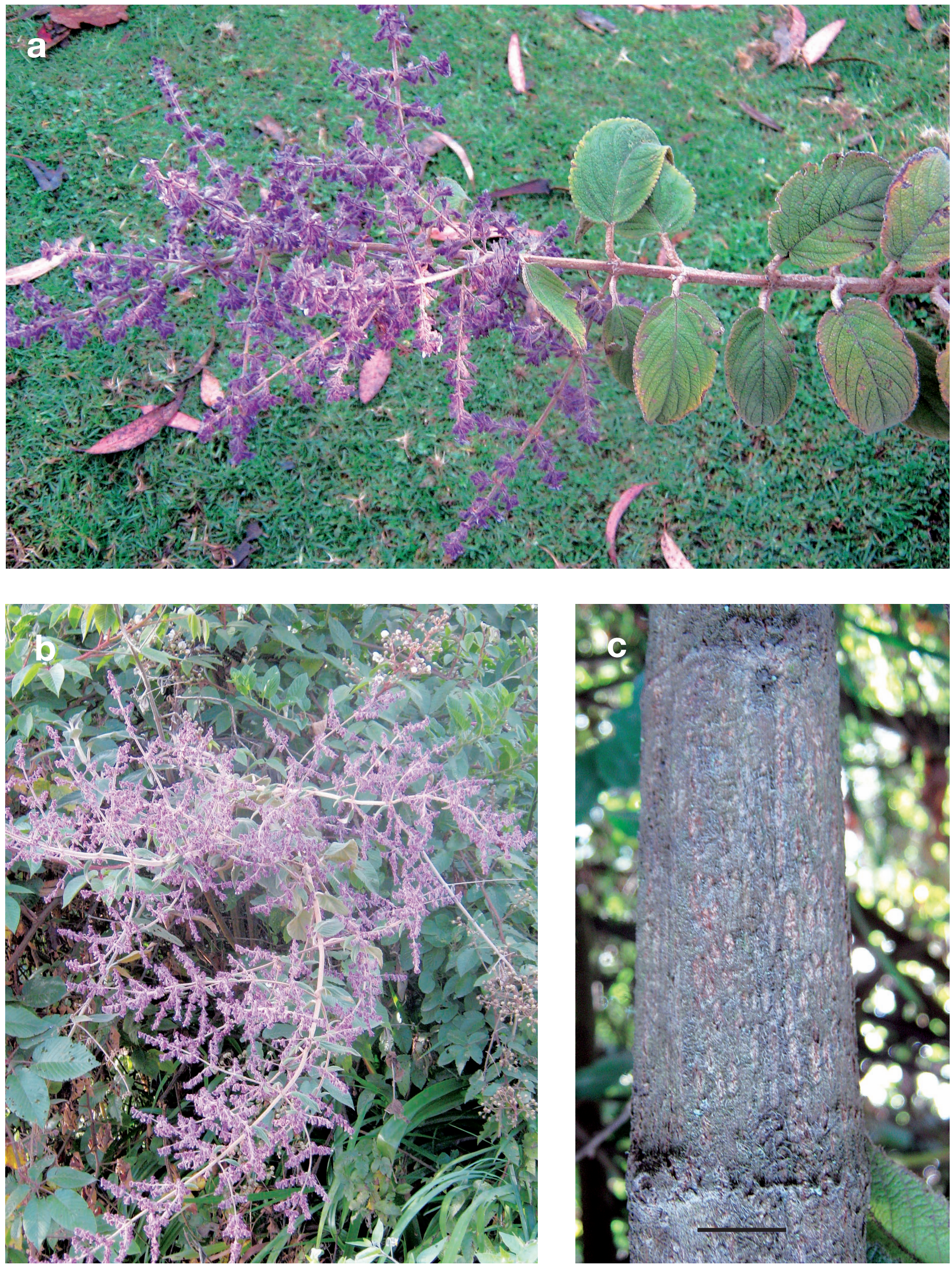

Fig. 2. Hyptis jacobi Fern. Alonso: a, eje terminal de la planta, mostrando la disposición dística de hojas e inflorescencia; b, típica panícula terminal ramosa y escandente en su posición natural; c, tronco de la planta a la altura del pecho, mostrando el indumento lanoso residual. Barra: 1,5 cm. (Fotografías que se corresponden con la serie típica, holótipo + isótipos, Fernández-Alonso \& al. 29193). 

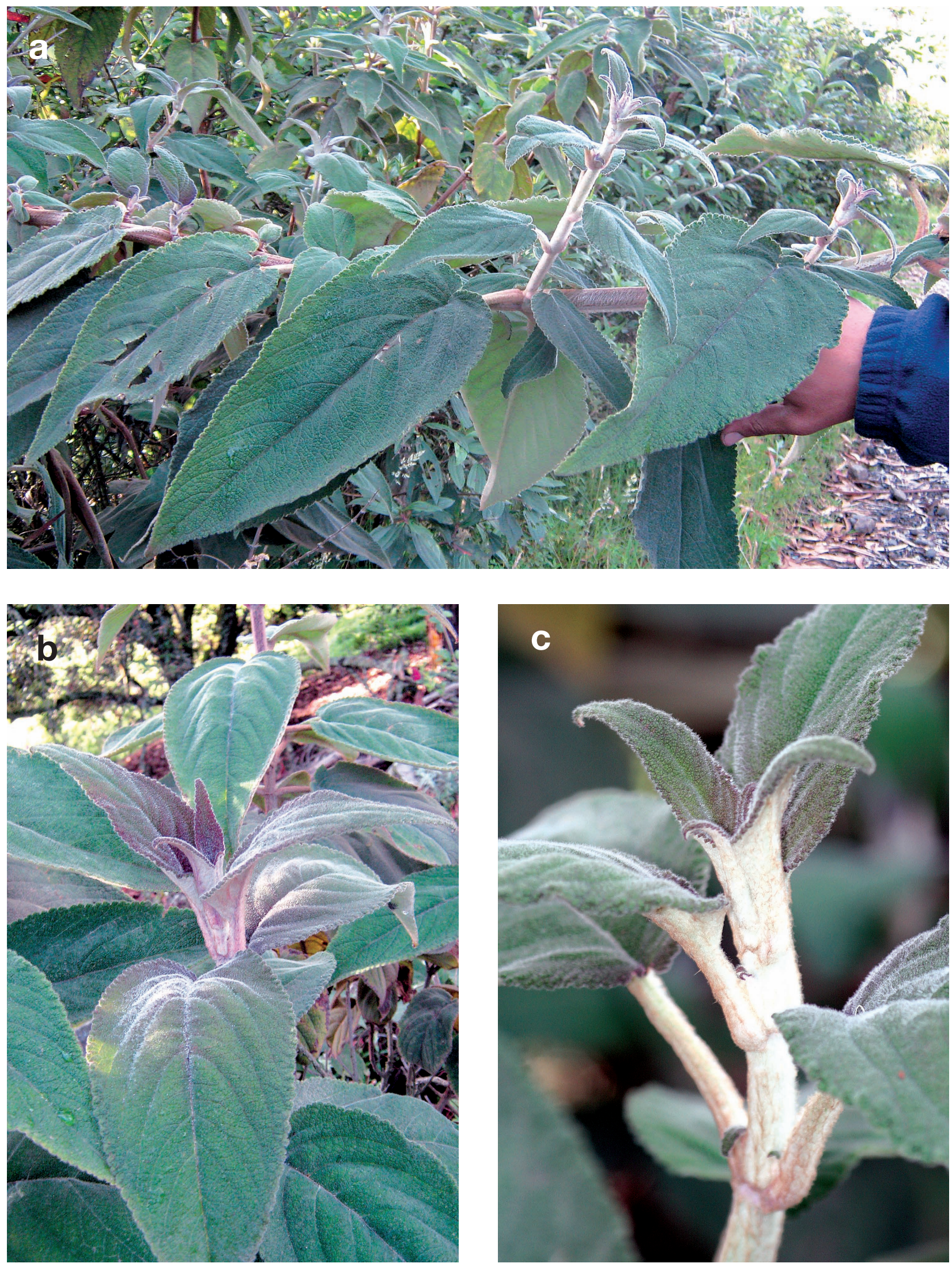

Fig. 3. Hyptis jacobi Fern. Alonso: a, rama vegetativa escandente; b, hojas jóvenes terminales con indumento morado; c, detalle del indumento lanoso en tallos, peciolos y envés foliar en los brotes jóvenes. (Fotografías que se corresponden con la serie de parátipos, Fernández-Alonso \& al. 29195). 

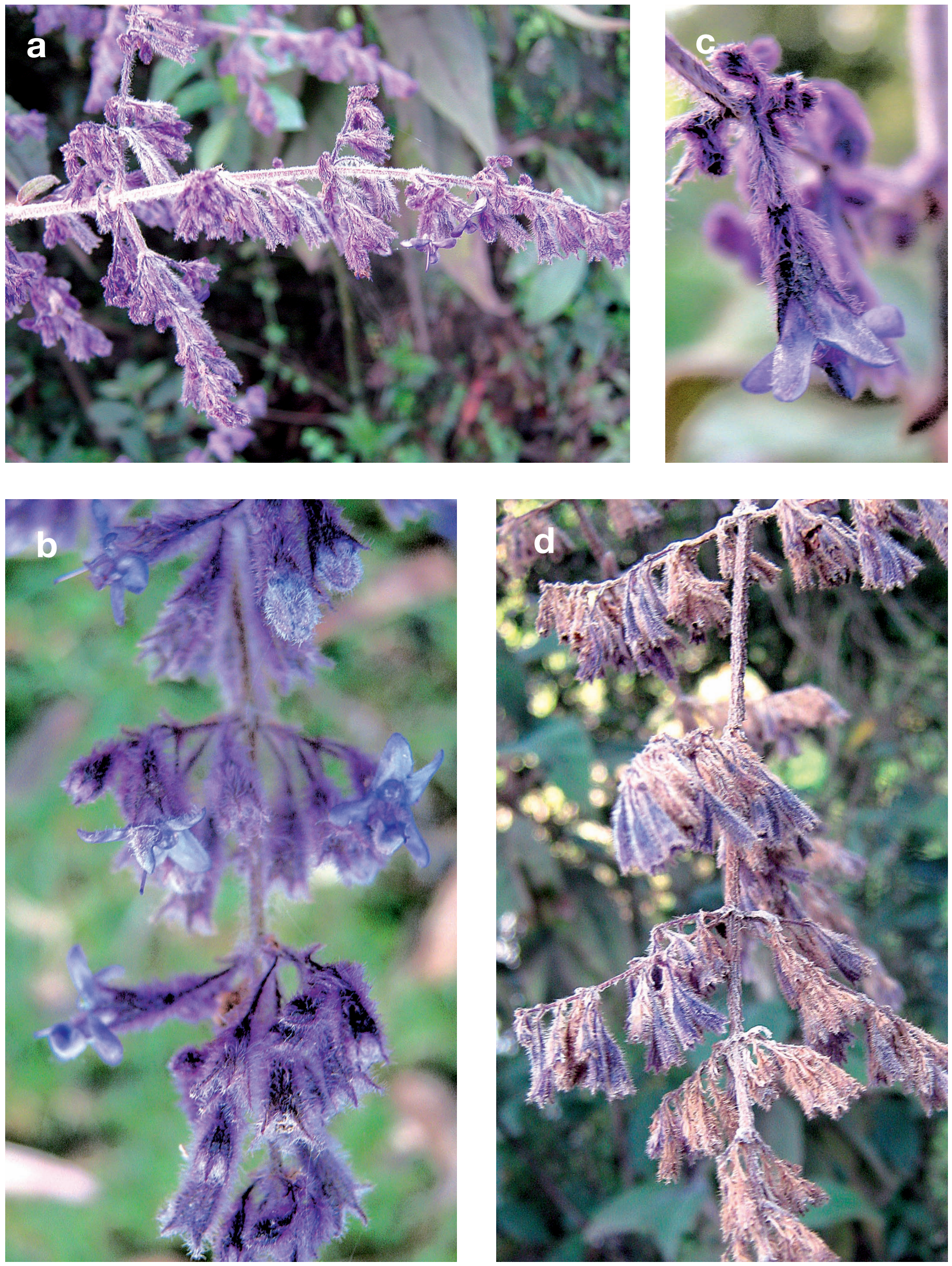

Fig. 4. Hyptis jacobi Fern. Alonso: a, b, ramificaciones de último orden en la panícula, mostrando las címulas; c, detalle de una flor y del indumento; d, cálices acrescentes al final de la floración. (Fotografías que se corresponden con la serie de parátipos, FernándezAlonso \& al. 29195). 

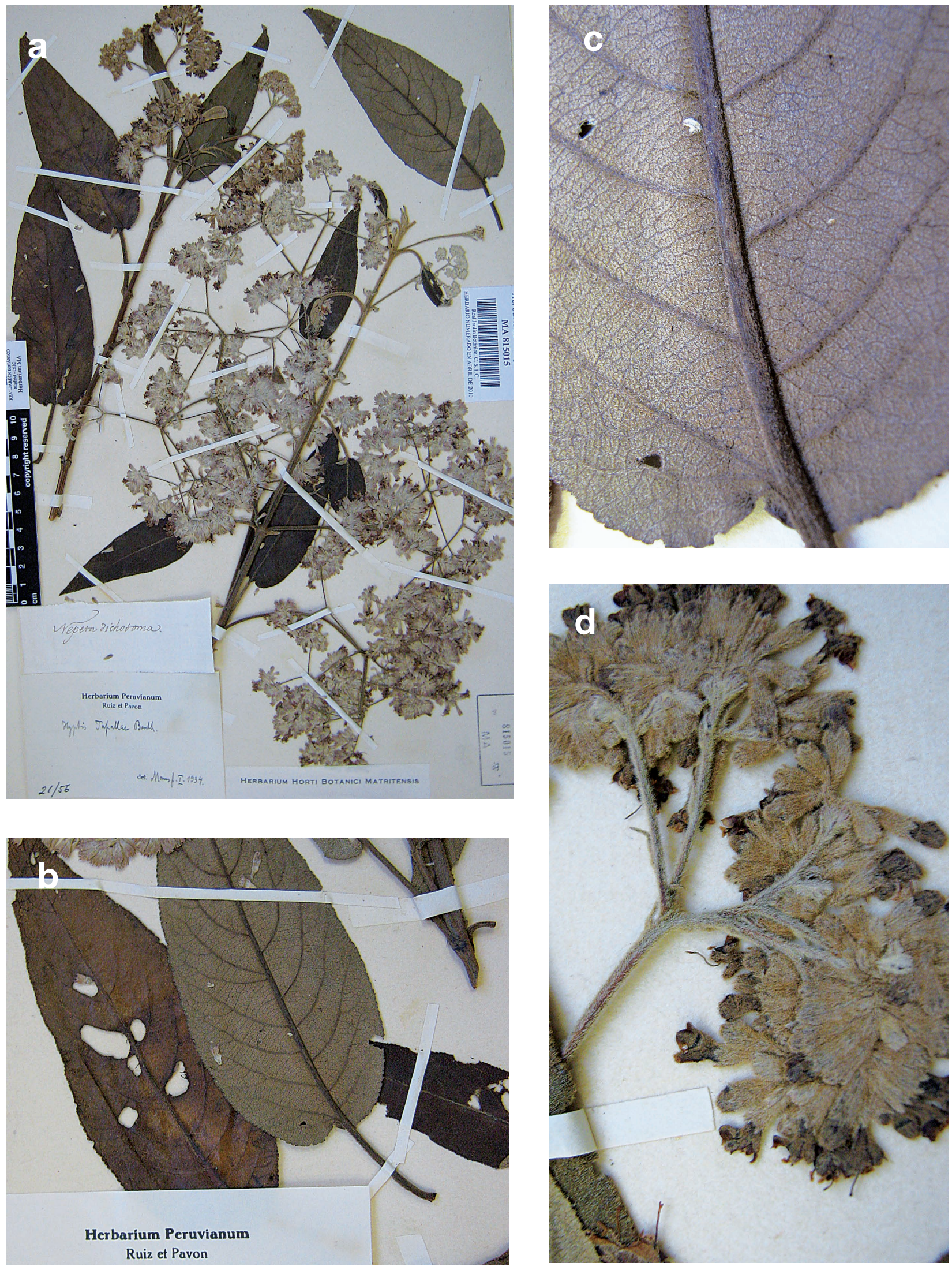

Fig. 5. Hyptis tafallae Benth.: a, pliego de la serie típica, de las colecciones originales del herbario de Ruiz \& Pavón, MA 801015; b, detalle de las hojas; $\mathbf{c}$, detalle del indumento en el envés; $\mathbf{d}$, detalle de las inflorescencias cimosas umbeliformes, largamente pedunculadas (herbario de Ruiz \& Pavón en MA). 
fitos (Blechnum L. Pteridium Gled. ex Scop.), Rosaceae (Rubus L.), Rubiaceae (Galium L.), Scrophulariaceae (Alonsoa Ruiz \& Pav., Calceolaria L.), Smilacaceae (Smilax L.), Solanaceae (Cestrum L., Solanum L.) y Verbenaceae (Duranta L., Lantana L.).

Por el reducido hábitat conocido de esta especie y el grado de alteración que presenta, consideramos prioritaria la evaluación detallada de su distribución y de su grado de amenaza. De acuerdo con los criterios actuales de categorización (IUCN, 2003; FernándezAlonso \& Rivera-Díaz, 2006) y sólo con la información conocida hasta ahora, consideramos en la actualidad esta especie en la categoría de Vulnerable (VU).

La especie que se describe, de acuerdo con un primer análisis, guarda afinidad con las especies de la sección Umbellatae (Epling) Epling, a la cual la adscribimos, sección que cuenta con al menos tres especies más de los Andes y de las montañas de México (Epling, 1949). De ellas es Hyptis tafallae Benth. de Perú y Bolivia (Macbride, 1960; Zarucchi, 1993) la especie más afín. De ella, no obstante, difiere claramente en el indumento ramoso y denso de los tallos e inflorescencias, en la disposición y tipo de inflorescencia sin umbelas largamente pedunculadas (Fig. $5 \mathrm{~d}$ ), en los dientes del cáliz más largos, de hasta $2 \mathrm{~mm}$, y en el menor tamaño del tubo de la corola, que no supera los 3,5 mm de longitud.

Aunque presenta también un notable parecido en el porte, indumento, inflorescencias y núculas con algunas especies de Hyptis sect. Budlejoides Benth., que posteriormente han sido consideradas dentro del género Hyptidendron Harley sect. Hyptidendron (Harley, 1988), su afinidad parece menos clara. A diferencia de la especie de Hyptis que se describe, Hyptidendron presenta hojas coriáceas, corolas de mayor tamaño con tubo de $6 \mathrm{~mm}$ o más y estilo provisto de estilopodio diferenciado por encima de las núculas. No obstante, los límites entre Hyptidendron y algunas secciones del género Hyptis, como es el caso de las secciones Umbellatae y Laniflorae Epling no parecen claramente establecidos en la actualidad. En este sentido apuntan también algunos análisis numéricos recientes (El-Gazzar \& Rabei, 2008).

Nombre vernáculo y usos: Según testimonios recogidos en la región, esta planta recibe el nombre de "Santa Marta" en su lugar de origen, donde es reputada como buen repelente para las moscas, y para ello se la coloca en las zonas de habitación.

\section{Parátipos}

COLOMBIA. Santander: En la vía Bucaramanga-Málaga, tramo Molagavita-Málaga, Pangote, $6^{\circ} 42^{\prime} \mathrm{N} 72^{\circ} 47^{\prime} \mathrm{W}, 2245$ m, 4-XII2006, arbusto apoyante de $3-4,5 \mathrm{~m}, \mathrm{~S}$. Albesiano E al. 2003, COL,
HUA, MA, UIS; ibídem, km 6-7 en la vía Málaga-Bucaramanga, después de Málaga, Vereda Buenavista, 642'29”N 7245’26”W, 2670 m, 29-VI-2009, arbusto de 4 m, fl, fr, J.L. Fernández-Alonso 28194 E C.N. Diaz-Pérez, COL, HUA, K, MA, SALA, UIS; ibídem, J.L. Fernández-Alonso 28195, 28197 E C.N. Diaz-Pérez, COL, MA, MEDEL.

\section{Material complementario revisado \\ de Hyptis tafallae Benth.}

PERÚ. Sin loc. s.f. Herb. Ruiz E Pavón s.n., MA 815015, MA 815014. Pasco: Prov. Oxapampa, road Oxapampa-Paucartambo, $1870 \mathrm{~m}$, roadside brush, 22-V-1982, D. Smith \& A. Pretel 1657, MO. Pro. Ayacucho, Huanta, 1700 m, I-1910, fl, 5608 A. Weberbauer (F 548493, typus de Hyptis tafalloides Mansf.).

BOLIVIA. Santa Cruz: Prov. Florida, 14 km of Bermejo, along road to Samaipata. Steep slopes of valley of Río Laja, $1250 \mathrm{~m}$, Shrub 2,4 m, 6-VIII-1987, fl., M. Nee E G. Coimbra 35539, MO.

\section{Agradecimientos}

A los curadores de los herbarios COL y MA, por facilitar el estudio de las muestras. A la Universidad Nacional de Colombia, por haber apoyado el trabajo de campo relacionado con los proyectos de prospección en labiadas. Al Ministerio de Ciencia e Innovación y al CSIC de España, por facilitar los trabajos de revisión que actualmente se llevan a cabo en plantas tropicales con cargo a los proyectos Intramural CSIC: 200930I071 y CGL2010-19747 (subprograma BOS). A Sofía Albesiano y Carlos N. Díaz, por su colaboración en la recolección de las muestras de esta especie. A Marcela Morales, por la excelente ilustración que acompaña este trabajo. A Ramón Morales y a dos revisores anónimos, por las valiosas observaciones al manuscrito.

\section{Referencias bibliográficas}

El-Gazzar, A. \& Rabei, S. 2008. Taxonomic assessment of five numerical methods and its implications on the classification of Hyptis s.l. (Labiatae). International Journal of Botany 4(1): 85-92.

Epling, C. 1949. Revisión del género Hyptis (Labiatae). Revista del Museo de La Plata, nov. ser., Sección Botánica 7: 153-497.

Fernández-Alonso, J.L. 1995. Estudios en Labiatae de Colombia I. Novedades en los géneros Salvia e Hyptis. Revista de la Academia Colombiana de Ciencias Exactas, Físicas y Naturales 19: 469-480.

Fernández-Alonso, J.L. 2008. Estudios en Labiatae VI - Salvia yukoyukparum, nueva especie y primer representante de la Sección Tomentellae en Colombia. Novon 18: 38-42.

Fernández-Alonso, J.L., Vega, N., Filgueira, J.J. \& Pérez, G. 2003. Lectin prospecting in Colombian Labiatae. A systematic-ecological approach. Biochemical Systematics and Ecology 31: 617-633.

Fernández-Alonso, J.L. \& Rivera-Díaz, O. 2006. Labiatae, pp. 385-582. In: García, N. \& Galeano, G. (eds.), Libro Rojo de Plantas de Colombia - 3. Serie Libros Rojos de especies amenazadas de Colombia. Instituto Alexander von Humboldt - Instituto de Ciencias Naturales de la Universidad Nacional de Colombia - Ministerio de Ambiente, Vivienda y Desarrollo Territorial. Bogotá.

Harley, R.M. 1988. Revision of generic limits in Hyptis Jacq. (Labiatae) and its allies. Botanical Journal of the Linnean Society 98: 87-95. 
Harley, R.M., Atkins, S., Budantsev, A.L., Cantino, P.D., Conn, B.J., Grayer, R., Harley, M.M., Kok, R. de, Krestovskaja, T., Morales, R., Paton, A.J., Ryding, O. \& Upson, T. 2004. Labiatae, pp. 167275. In: Kubitzki, K. (ed. in chief) \& Kadereit,J.W. (ed.), The families and genera of vascular plants. VII. Flowering plants. Dicotiledons: Lamiales (except Acanthaceae including Avicenniaceae).

IUCN. 2003. Directrices para emplear los criterios de la Lista Roja de la IUCN a nivel nacional y regional. Versión 3.0. Preparados por la Comisión de supervivencia de Especies de la UICN. IUCN Unión Mundial para la Naturaleza.
Macbride, J.F. 1960. Lamiaceae. En Flora of Perú. Field Museum Natural History Botanical Series 13, 5(2): 721-829.

Zarucchi, J.L. 1993. Lamiaceae, pp. 579-590. In: Brako, L. \& Zarucchi, J.L. (eds), Catálogo de las Angiospermas y Gimnospermas del Perú. Mongraphs of Systematic Botany of the Missouri Botanical Garden 45. St. Louis.

Editor asociado: R. Morales Recibido: 22-VII-2010 Aceptado: 22-X-2010 